\title{
Correction to: Neglected Elements of a High-Quality Early Childhood Workforce: Whole Teacher Well-Being and Working Conditions
}

\author{
Kyong-Ah Kwon ${ }^{1} \cdot$ Timothy G. Ford $^{1} \cdot$ Alicia L. Salvatore $^{2} \cdot$ Ken Randall $^{3} \cdot$ Lieny Jeon $^{4} \cdot$ Adrien Malek-Lasater $^{1}$. \\ Natalie Ellis ${ }^{5}$. Mia S. Kile ${ }^{5}$. Diane M. Horm ${ }^{6}$. Sun Geun Kim ${ }^{1} \cdot$ Minkyung Han ${ }^{1}$
}

Published online: 5 December 2020

(c) Springer Nature B.V. 2020

\section{Correction to: Early Childhood Education Journal https://doi.org/10.1007/s10643-020-01124-7}

The original version of this article unfortunately contains some typo in Table 1.
In Table 1, the values of the columns "Full sample" and "By age group" were incorrect because the digits should be in 10 s.

The correct Table 1 is presented here.

The original article has been corrected.

The original article can be found online at https://doi.org/10.1007/ s10643-020-01124-7.

Kyong-Ah Kwon

kasdream@gmail.com

1 The University of Oklahoma-Tulsa, 4502 E. 41st Street

(Suite 4W120), Tulsa, OK 74135, USA

2 Value Institute, ChristianaCare, Newark, DE, USA

3 The University of Oklahoma Health Science Center, Oklahoma City, OH, USA

4 Johns Hopkins University, Baltimore, MD, USA

5 The University of Oklahoma, Norman, OH, USA

6 Early Childhood Education Institute, The University of Oklahoma-Tulsa, Oklahoma, USA 
Table 1 Demographic characteristics and working conditions for 262 ECE teachers (mean or \%)

\begin{tabular}{|c|c|c|c|c|c|c|c|}
\hline \multirow[t]{2}{*}{ Categories } & \multirow[t]{2}{*}{ Full sample (range) } & \multicolumn{3}{|c|}{ By age group } & \multicolumn{3}{|c|}{ By education level } \\
\hline & & $\begin{array}{l}\text { I-T teacher } \\
(\mathrm{n}=158)\end{array}$ & $\begin{array}{l}\text { Preschool } \\
\text { teacher } \\
(\mathrm{n}=104)\end{array}$ & $p$-value & $\begin{array}{l}\text { With BA } \\
\text { degree } \\
(\mathrm{n}=105)\end{array}$ & $\begin{array}{l}\text { W/O BA } \\
\text { degree } \\
(n=157)\end{array}$ & $p$-value \\
\hline \multicolumn{8}{|l|}{ Demographic characteristics } \\
\hline Age & $36.6(18-66)$ & 36.6 & 36.6 & .98 & 37.5 & 36.0 & .31 \\
\hline \multicolumn{8}{|l|}{ Race/ethnicity } \\
\hline African American & $22.0 \%$ & $22.0 \%$ & $23.0 \%$ & .73 & $16.2 \%$ & $26.3 \%$ & .05 \\
\hline Caucasian & $56.0 \%$ & $50.0 \%$ & $66.0 \%$ & $<.01$ & $65.7 \%$ & $50.0 \%$ & .01 \\
\hline Hispanic & $7.0 \%$ & $9.0 \%$ & $2.0 \%$ & $<.01$ & $4.8 \%$ & $7.7 \%$ & .34 \\
\hline Native American & $10.0 \%$ & $12.0 \%$ & $6.0 \%$ & .07 & $6.7 \%$ & $12.2 \%$ & .15 \\
\hline Asian & $2.0 \%$ & $3.0 \%$ & $0.0 \%$ & .11 & $2.9 \%$ & $0.6 \%$ & .15 \\
\hline Biracial & $3.0 \%$ & $4.0 \%$ & $3.0 \%$ & .70 & $3.8 \%$ & $3.2 \%$ & .79 \\
\hline \multicolumn{8}{|l|}{ Education } \\
\hline High school or less & $16.0 \%$ & $21.0 \%$ & $9.0 \%$ & $<.01$ & - & - & \\
\hline Associate degree/some college & $44.0 \%$ & $44.0 \%$ & $43.0 \%$ & .86 & - & - & \\
\hline BA degree or higher & $40.0 \%$ & $35.0 \%$ & $48.0 \%$ & $<.05$ & - & - & \\
\hline Experience in education (years) & $11.9(0-40)$ & 11.7 & 12.1 & .67 & 12.1 & 11.7 & .72 \\
\hline \multicolumn{8}{|l|}{ Early head start/head start } \\
\hline Yes & $43.0 \%$ & $41.0 \%$ & $45.0 \%$ & .54 & $64.8 \%$ & $28.0 \%$ & $<.00$ \\
\hline \multicolumn{8}{|l|}{ Job position } \\
\hline Lead teacher & $61.0 \%$ & $59.0 \%$ & $64.0 \%$ & .36 & $82.9 \%$ & $46.5 \%$ & $<.00$ \\
\hline Assistant teacher & $39.0 \%$ & $41.0 \%$ & $36.0 \%$ & - & $17.1 \%$ & $53.5 \%$ & -- \\
\hline \multicolumn{8}{|l|}{ Working conditions } \\
\hline Environmental Preference Index & $.80(0-1)$ & .81 & .80 & .42 & .80 & .81 & .41 \\
\hline \multicolumn{8}{|l|}{ Space for relaxation } \\
\hline Yes & $67.0 \%$ & $66.0 \%$ & $70.0 \%$ & .53 & $65.4 \%$ & $68.6 \%$ & .59 \\
\hline \multicolumn{8}{|l|}{ Space for personal belonging } \\
\hline Yes & $69.0 \%$ & $68.0 \%$ & $70.0 \%$ & .83 & $68.3 \%$ & $69.2 \%$ & .87 \\
\hline \multicolumn{8}{|l|}{ Noise disturbing } \\
\hline Yes & $74.0 \%$ & $77.0 \%$ & $70.0 \%$ & .21 & $73.1 \%$ & $74.4 \%$ & .82 \\
\hline \multicolumn{8}{|l|}{ Furniture not adult-sized } \\
\hline Yes & $19.0 \%$ & $20.0 \%$ & $18.0 \%$ & .60 & $23.1 \%$ & $16.7 \%$ & .20 \\
\hline \multicolumn{8}{|l|}{ Designated break } \\
\hline Yes & $56.0 \%$ & $64.0 \%$ & $45.0 \%$ & $<.00$ & $40 \%$ & $67.5 \%$ & $<.00$ \\
\hline \multicolumn{8}{|l|}{ Additional staff } \\
\hline Yes & $90.0 \%$ & $89.0 \%$ & $93.0 \%$ & .38 & $94.2 \%$ & $84.1 \%$ & $<.05$ \\
\hline Perceived work environment & $3.5(1-5)$ & 3.5 & 3.4 & .45 & 3.5 & 3.5 & \\
\hline \multicolumn{8}{|l|}{ Salary } \\
\hline$\$ 20,000$ or less & $29.0 \%$ & $29.0 \%$ & $29.0 \%$ & .95 & $11.5 \%$ & $41.0 \%$ & $<.00$ \\
\hline$\$ 20,001-\$ 40,000$ & $55.0 \%$ & $56.0 \%$ & $54.0 \%$ & .82 & $52.9 \%$ & $57.7 \%$ & .44 \\
\hline$\$ 40,001$ or more & $16.0 \%$ & $15.0 \%$ & $16.0 \%$ & .80 & $35.6 \%$ & $1.3 \%$ & $<.00$ \\
\hline \multicolumn{8}{|l|}{ Health insurance } \\
\hline Yes & $69.0 \%$ & $65.0 \%$ & $76.0 \%$ & .05 & $82.9 \%$ & $59.9 \%$ & $<.00$ \\
\hline \multicolumn{8}{|l|}{ Paid vacation days } \\
\hline Yes & $75.0 \%$ & $77.0 \%$ & $72.0 \%$ & .35 & $81.0 \%$ & $71.3 \%$ & .08 \\
\hline \multicolumn{8}{|l|}{ Paid sick days } \\
\hline Yes & $61.0 \%$ & $61.0 \%$ & $62.0 \%$ & .90 & $73.3 \%$ & $52.9 \%$ & $<.00$ \\
\hline \# of paid vacation/sick days & 12.5 & 12.2 & 12.9 & .49 & $14.6 \%$ & $11.1 \%$ & $<.00$ \\
\hline \multicolumn{8}{|l|}{ Retirement plan provided } \\
\hline Yes & $58.0 \%$ & $57.0 \%$ & $60.0 \%$ & .64 & $75.0 \%$ & $46.5 \%$ & $<.00$ \\
\hline
\end{tabular}


Table 1 (continued)

\begin{tabular}{|c|c|c|c|c|c|c|c|}
\hline \multirow[t]{2}{*}{ Categories } & \multirow[t]{2}{*}{ Full sample (range) } & \multicolumn{3}{|c|}{ By age group } & \multicolumn{3}{|c|}{ By education level } \\
\hline & & $\begin{array}{l}\text { I-T teacher } \\
(\mathrm{n}=158)\end{array}$ & $\begin{array}{l}\text { Preschool } \\
\text { teacher } \\
(\mathrm{n}=104)\end{array}$ & $p$-value & $\begin{array}{l}\text { With BA } \\
\text { degree } \\
(n=105)\end{array}$ & $\begin{array}{l}\text { W/O BA } \\
\text { degree } \\
(n=157)\end{array}$ & $p$-value \\
\hline \multicolumn{8}{|l|}{ Well-being as priority } \\
\hline Yes & $74.0 \%$ & $77.0 \%$ & $70.0 \%$ & .20 & $72.4 \%$ & $75.8 \%$ & .54 \\
\hline \multicolumn{8}{|c|}{ Resources for well-being } \\
\hline Yes & $70.0 \%$ & $72.0 \%$ & $65.0 \%$ & .25 & $72.4 \%$ & $67.5 \%$ & .40 \\
\hline Job stressors & $2.7(1-5)$ & 2.6 & 2.9 & $<.00$ & 2.73 & 2.7 & .48 \\
\hline Physical job demand & $3.3(1-5)$ & 3.4 & 3.1 & $<.00$ & 3.3 & 3.2 & .78 \\
\hline
\end{tabular}

Pearson chi-square test used for dichotomous variables

Publisher's Note Springer Nature remains neutral with regard to jurisdictional claims in published maps and institutional affiliations. 